\title{
A Laboratory Study of the Sound-Odour Interaction in Urban
}

\author{
Environments
}

\author{
Meihui $\mathrm{Ba}^{\mathrm{a}}$, Jian Kang ${ }^{\mathrm{a}, \mathrm{b}^{*}}$
}

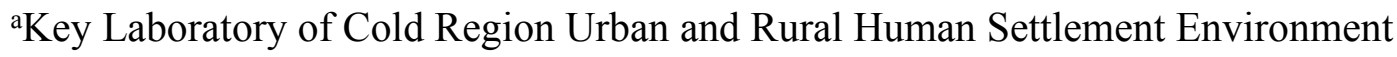
Science and Technology, Ministry of Industry and Information Technology, School of Architecture, Harbin Institute of Technology, Harbin, China.
bUCL Institute for Environmental Design and Engineering, University College London (UCL), London, United Kingdom.

*Corresponding Author: J.kang@ucl.ac.uk, UCL Institute for Environmental Design and Engineering, The Bartlett, University College London (UCL), London WC1H 0NN, United Kingdom. Tel: +44 (0)20 31087338.

Received: 18 July 2018 Revised: 9 October 2018 Accepted: 10 October 2018 Available online: 11 October 2018

\begin{abstract}
This study investigated the interaction of sound and odour in urban environments. Sound and odour perception as well as overall perception were evaluated by 168 participants through a laboratory experiment, where different sounds and odours were selected, each with three levels. The results showed that, in the evaluation of sound, odour had little effect on evaluations of birdsong and low-volume sound, whereas for the rest of the conditions, higher odour concentration resulted in a more positive evaluation. The odour evaluation showed that, with increase of volume, odour evaluation became more negative, and for such results traffic and conversation sounds had the greatest effects. For the overall evaluation, in the presence of birdsong and low-volume sound, the overall evaluation was unaffected by odour; for other combinations of sound and odour, with increased concentration, the overall evaluation improved. Moreover, the similarities and differences in the evaluations of sound and odour showed an analogous trend of sensory comfort and preference due to their high correlation coefficient-a positive sensory stimulus can improve the evaluation of other senses, while a negative one has the opposite effect. There is a masking effect between audition and olfaction that is reflected in the finding that when one stimulus is stronger, the other has weaker perceptual intensity; however, sensory familiarity does not change. Furthermore, the relationship between sensory evaluation and overall evaluation showed that for overall comfort, the effect of sound was stronger than odour, while for congruency, the effects of sound and odour were almost equal.
\end{abstract}

Keywords: sound; odour; audio-olfactory interaction; multisensory evaluations 


\section{Introduction}

In our contemporary urban environments, both physical design and psychological design are necessary, given improvements in people's living standards [1]. The senses include vision, audition, olfaction, taste and touch [2]. In experiencing the outside world, the senses of vision, audition, and olfaction have the opposite characteristics to the senses of taste and touch. Vision, audition, and olfaction often arise from unconscious behaviour and cannot always be avoided at the beginning of an experience; taste and touch are more dependent on people's subjective will [3]. Taste and touch are somewhat limited in attempting to grasp the outside ambient; vision together with audition and olfaction are important media for perceiving the environment. Multi-sense is capable of transmitting more profound and comprehensive information than single sense perception, and it is also more capable of meeting the needs of the human brain in integrating and perceiving the external world through different sensory channels [4].

At present, research on sensory interaction is mainly focused on audio-visual interaction. Studies have shown that vision and audition can affect each other. Southworth reported that in an audio-visual environment, attention to visual landscape leads to a decline in the sense of audition and vice versa [5]. In terms of the effects of visual stimulation on auditory perception, Carles et al. found that the more urban a landscape is, the more complex the auditory sensation associated with it [6]. Viollon found that visual parameters had more obvious influence on auditory judgement in the integrated audio-visual environment [7]. D'Alessandro et al.'s study, which was carried out in a university external area, found that the difference in perception of sound and the overall environment between winter and summer is mainly attributed to a higher human voice component, more pleasant visual aspects, and climate conditions during the summer [8]. Maffei et al. chose a wind farm located in a quiet area as a research setting and used virtual reality to study the effect of visual factors (e.g. distance, quantity, and colour of turbines) on the degree of noise acceptance. The results showed that the evaluation of noise was better when wind turbines were far away, fewer in quantity, and green or white in colour [9]. Ren and Kang found that visual factors of waterscape, including landscape type, distance from people, and the appearance of animals and humans, could affect acoustic comfort [10]. Liu et al. analysed the effects of physical characteristics of visual landscape on soundscape perception in city parks through a designed soundwalk method [11]. The results suggest that the percentage of buildings, vegetation, and sky in panoramic views were effective landscape elements influencing soundscape perception. In the study of Carles et al. [12], the testers were asked to evaluate the images and sounds of 36 natural and semi-natural spaces in cities. The results showed that the addition of a visual landscape to the act of overall perception of the environment had two effects on audition: it increased recognition and enhanced semantics. As for the effects of auditory stimulation on visual perception, Mace et al. found that aircraft noise had such a negative impact on the perception of the scenery within natural parks that it could change the perceiver's evaluation of the scenery [13]. Lindquist et al. found that the sense of reality was higher when sound and visual scene were congruent through $3 \mathrm{D}$ simulation [14].

Visual-olfactory interaction has also been the centre of study. Van Reekum et al. selected abstract paintings, sounds and odours to represent visual, audio, and olfactory stimuli [15]. Sounds and odours that people liked and those that people hated were added to paintings of neutral evaluation in the experiment. The results indicated that people's evaluation of vision was 
influenced by audio and olfactory factors. Dinh et al. found that adding sensory factors such as odour could increase people's memory of a place, whereas the addition of visual detail did not [16]. Studies also showed that visual information can affect odour perception [17-19]. On the other hand, odour can also influence visual perception [20-23]. So far, the study of the interaction between audition and olfaction has been limited. Michon et al. found that music and fragrance could affect shopping behaviour in shopping malls [24]. Consumer spending was significantly higher when arousing music and ambient odours were congruent, while incongruent sensory stimuli had a contrary result. Jiang et al. investigated the influence of odour and vision on people's evaluation of road traffic, with results showing that the presence of odour had no effect on evaluation but that odour had significant interaction with traffic noise level and the effect of trees on landscape quality [25]. Jiang et al.'s experiment demonstrated the potential of odour to regulate noise and landscape perception in a specific environment.

This study aimed to discern the sense perception regularity of the interaction between audition and olfaction in urban environments. The research methods of sensory interaction mainly included field research and laboratory experimentation. Field research is more immersive in the midst of an active environment in which many stimuli may coexist [24,26,27], whereas laboratory experiment allows for more accurate control over sensory stimuli $[28,29]$. The laboratory research approach was utilised in this study. Previous studies usually chose only one type of sensory stimulus, and most had only two conditions (present or absent). Therefore, this study investigated two types of sensory stimuli (sounds and odours) and two types of conditions (volume and concentration), in an attempt to be more systematic in our investigation and to obtain more concrete results. Three research questions were addressed in the study: (1) "Does odour affect the perceiver's evaluation of sound?" (2) "Does sound affect the perceiver's evaluation of odour?" (3) "Do sound and odour affect the overall evaluation?"

\section{Method}

\subsection{Overall experimental design}

The experimental results are to be applied to outdoor environments, so three types of sounds and four types of odours that are common sensory sources in urban environments were selected in the experiment. The volume of sound and the concentration of odour were both controlled to three levels: low, medium and high.

Therefore, there were nine conditions of experiments with only sound $(3 \times 3), 12$ conditions with only odour $(4 \times 3)$, and 108 conditions with both sound and odour $(3 \times 3 \times 4 \times 3)$. In total, 129 conditions of full factorial experimental design were made to present all possible combinations of the factors.

\subsection{Odour stimuli}

For odour stimuli, we considered what could be made available and safely controlled within a laboratory setting. Sources of pollution odour are harmful and often cannot be easily controlled. We instead decided to focus upon plant and food odours, which were generally safer and easier to 
control in experiments than pollution sources. Among them, lilac and osmanthus odours were chosen for plant odours, as both are from common varieties of urban greening. Coffee and bread odours were chosen for food odours, because they are single, unmixed odours as opposed to the varying, mixed and changeable odours of more complex foods, including cooked dishes, often found at restaurants and markets.

Essential oils and perfume whose ingredients were extracted from natural sources were chosen as odour sources because it facilitated accurate dilution so that samples of different concentrations could be obtained for testing while avoiding concomitant visual interference. To ensure that the selected essential oil and perfume could replace the real odour, a pre-experiment on the authenticity of the odour stimuli was carried out in the laboratory. The evaluation of authenticity must be based on the familiarity of the participant with the odour; thus, a pre-experiment on the familiarity rating of odour stimuli was carried out. Thirty participants who were familiar with the four odours indicated above were selected to evaluate the authenticity of the odour (1-very unreal, 2-unreal, 3-moderate, 4-real, 5-very real). The essential oil and perfume source with the highest mean ratings for authenticity and over 4 were selected as odour sources in this experiment.

The concentration of odour stimuli was controlled to three levels: low, medium and high. The intensity rating of odour stimuli was also included in the pre-experiment mentioned above. Liquid odour sources (essential oil and perfume) were diluted into different concentration gradients, the subjects were selected to evaluate the subjective intensity (1-very light, 2-light, 3-moderate, 4-strong, 5-very strong), and then the dilution ratios, which corresponded to the evaluations of light, moderate and strong, were selected as the concentrations for odour stimuli. In this study, the liquid odour sources were extracted with a syringe, which facilitated easy measurement, and the dilution of concentration gradient was adjusted by adding distilled water [30]. Then the mixed liquid was sprayed evenly on the medical tampon in a beaker with an atomizer. The volume ratios of odour source liquid and distilled water corresponded to the concentrations of low, medium and high as shown in Table 1.

Table 1 Volume ratio setting of odour sources liquid and distilled water

\begin{tabular}{ccccc}
\hline Concentration & Lilac & Osmanthus & Coffee & Bread \\
\hline Low & $1: 5$ & $1: 5$ & $1: 10$ & $1: 10$ \\
Medium & $2: 5$ & $2: 5$ & $1: 5$ & $1: 5$ \\
High & $4: 5$ & $4: 5$ & $2: 5$ & $2: 5$ \\
\hline
\end{tabular}

\subsection{Audio stimuli}

For audio stimuli, the sounds of birds, conversation, and traffic were selected, corresponding to natural, anthropogenic and mechanical sounds, respectively, which are common in urban environments. The sound recording was carried out in the field by using a 10-channel portable recorder in hi-fidelity. The recording equipment was placed at a height of 1.5 metres perpendicular to the ground, with each recording lasting for a duration of five minutes. At the same time, the immediate surrounding was recorded through the video function of the digital camera (Nikon N1), to compare and analyse the time and position of both special and disturbing sound sources. Take traffic sound as an example: sounds from lorries, buses, motorbikes, and any vehicles other than 
cars were excluded from the selection to avoid dramatic differences in frequency spectrum across the samples [25].

A representative 40-second sample recording at each location was edited as an audio stimulus. Sometimes a sound could appear to be too loud and could cause discomfort when the laboratory simulated the same sound pressure level as in the field; this is due to the difference between their background sound pressure level. Therefore, the actual sound pressure level was weakened in the laboratory experiment and was calibrated and measured by an HMS IV artificial head to simulate a human ear. The adjustment of sound pressure level was processed by Adobe Audition software. With $10 \mathrm{~dB}$ as the gradient of sound pressure level, the pre-experiment mentioned in Section 2.2 also included the selection of sound volume. Subjects were asked to evaluate the subjective loudness (1-very quiet, 2-quiet, 3-moderate, 4-noisy, 5-very noisy). Then the sound pressure level, which corresponded to the evaluation of quiet, moderate and noisy, was selected as the volume level for each audio stimulus. The volume of each audio stimulus corresponded to low, medium and high as shown in Table 2 .

Table 2 Audio stimuli parameters

\begin{tabular}{|c|c|c|c|c|c|c|}
\hline \multirow{2}{*}{ Sound source } & \multirow{2}{*}{ Volume } & \multicolumn{5}{|c|}{ A-weighted sound pressure level (dB) } \\
\hline & & LA,eq & LA,max & LA,min & $\mathrm{LA}, 10$ & LA,90 \\
\hline \multirow{3}{*}{ Bird } & Low & 38.5 & 42.5 & 32.7 & 43.3 & 28.4 \\
\hline & Medium & 48.4 & 52.6 & 41.0 & 53.0 & 32.2 \\
\hline & High & 58.0 & 62.3 & 50.2 & 63.0 & 40.9 \\
\hline \multirow{3}{*}{ Conversation } & Low & 40.2 & 42.3 & 38.1 & 42.6 & 36.5 \\
\hline & Medium & 49.9 & 52.2 & 46.8 & 52.6 & 45.7 \\
\hline & High & 59.9 & 62.2 & 56.9 & 62.5 & 55.8 \\
\hline \multirow{3}{*}{ Traffic } & Low & 39.8 & 42.9 & 36.8 & 42.5 & 35.3 \\
\hline & Medium & 49.4 & 52.7 & 45.9 & 52.2 & 44.5 \\
\hline & High & 59.4 & 62.7 & 55.9 & 62.3 & 54.4 \\
\hline
\end{tabular}

\subsection{Questionnaire design}

Questionnaire distribution was conducted in the laboratory to investigate the subjects' evaluation, mainly from the following three aspects which corresponded to the three research questions. Each part has its own evaluation indicators, as shown in Fig. 1.

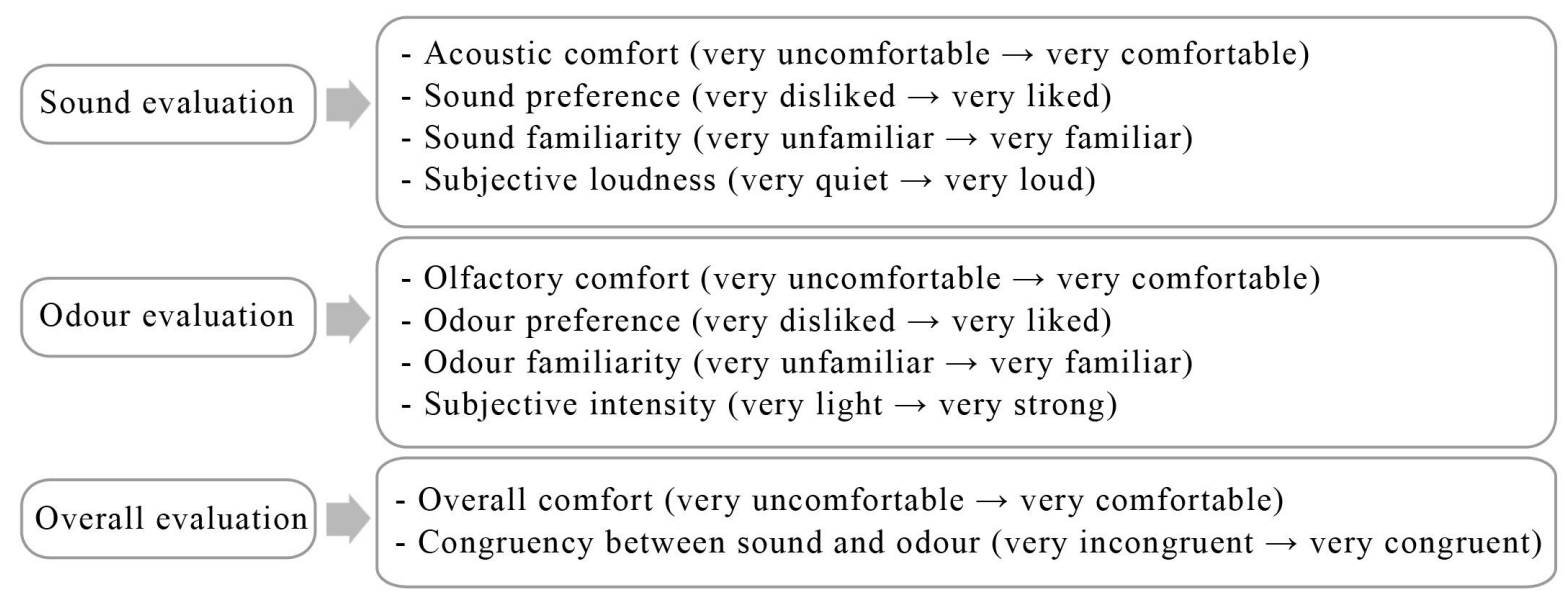

Fig. 1 Contents of questionnaire and specific evaluation indicators 
A 5-point Likert scale was used as follows: very uncomfortable, uncomfortable, moderate, comfortable, and very comfortable, with corresponding scores 1 to 5 . The reliability and validity of the questionnaires were tested before statistical analysis.

\subsection{Participants}

Previous studies examining the perception of multisensory stimuli in a lab setting used as few as 20 participants [31]. In our study 168 participants (54.8\% females) including $42.9 \%$ undergraduates and $57.1 \%$ postgraduates, average age 22 ( $\mathrm{SD}=2.6$; Min=18; Max=27), with self-reported normal audition and olfaction, no mental illness, and not pregnant [32], were recruited for the experiment without payment. All participants were recruited from universities via Internet and by personal contacts.

\subsection{Experimental procedure}

The experiment was carried out in a sound insulation chamber, the walls of which were equipped with sound absorbing materials and structures, so as to reduce reverberation and background noise within the room. Aside from the necessary experimental equipment, no other objects interfered with attention [33]. The audio stimuli were played through five loudspeakers, thus forming the effect of stereo sound and maximising the restoration of live recording. An RME FireFace UFX sound card and Genelec audio system (including front middle speaker: 1038BC, front left and right speakers: 1038B, back left and right subwoofers: 1037C) were used. The subjects were placed in the middle of the room. To avoid the effect of hunger on the perception of food odour, the experiment time was scheduled from 9:00 to 11:00 and 13:00 to 15:00. During the experiment, the door was closed and the temperature was controlled at $25^{\circ} \mathrm{C}$. No perfume, smoking, or other factors affected the results [34]. The participants underwent the experiment individually. One researcher arranged the experimental equipment and gave a brief introduction of the experimental procedure to the participant. When the experiment started, the researcher left the room without disturbing the participant. The specific experimental flow was as follows. (1) In the sound evaluation segment, 9 audio stimuli were played in a random sequence for 40 seconds. The interval between each stimulus lasted 10 seconds. The subjects filled in the questionnaire during each sound playing period and interval. (2) In the odour evaluation segment, 12 odours were given randomly. A beaker containing the odour source was placed on the desk and directly under the nose of the participant at a distance of $30 \mathrm{~cm}$. A $30 \mathrm{~cm}$ scale was fixed on the side of the beaker for reference. The subjects were asked to smell each given odour for 40 seconds, and then to fill in the corresponding part in the questionnaire. (3) In the overall evaluation segment, a combination of sound and odour stimuli were given together randomly. The subjects also had 40 seconds to fill in the questionnaires. After each combination was evaluated, the room was ventilated for five minutes. A ventilator was used to accelerate the process. The subjects waited and took deep breaths outside the laboratory to clear the substances from their nasal cavity. After ventilation, the subjects re-entered the laboratory and continued with the experiment. Each odour stimulus was newly prepared for different subjects. 


\section{Results}

\subsection{Sound evaluation}

\subsubsection{The effect of odour stimuli}

IBM SPSS Statistics 21.0 was used to establish a database with all results [35]. Sound evaluations without odour stimuli are shown in Table 3. In terms of acoustic comfort, there was a positive attitude toward natural sound in this study, while the opposite was true for mechanical sound [12,36,37]. Specifically, the highest mean rating for acoustic comfort occurred when the birdsong was played at medium volume. The acoustic comfort of conversation and traffic sounds declined with increased volume. The trend for sound preference was similar to that of acoustic comfort; that is, sound with high acoustic comfort also had high preference, which was consistent with previous studies [38,39]. For sound familiarity, the mean ratings were all high and over 4. Finally, for subjective loudness, whatever the sound, the ratings were quite close no matter whether the volume was low, medium or high. This is consistent with the aim that taking the sound pressure levels which corresponded to the subjective evaluation of quiet, moderate, and noisy as the volumes for sound stimuli in Section 2.3.

Table 3 Sound and odour evaluations without their mutual effects

\begin{tabular}{|c|c|c|c|c|c|c|c|c|c|c|}
\hline \multirow{2}{*}{$\begin{array}{l}\text { Sensory } \\
\text { stimuli }\end{array}$} & \multirow{2}{*}{$\begin{array}{l}\text { Sensory } \\
\text { source }\end{array}$} & \multirow{2}{*}{$\begin{array}{c}\text { Volume } \\
\text { (Concentration) }\end{array}$} & \multicolumn{2}{|c|}{ Sensory comfort } & \multicolumn{2}{|c|}{$\begin{array}{l}\text { Sensory } \\
\text { preference }\end{array}$} & \multicolumn{2}{|c|}{$\begin{array}{l}\text { Sensory } \\
\text { familiarity }\end{array}$} & \multicolumn{2}{|c|}{$\begin{array}{c}\text { Subjective } \\
\text { loudness (intensity) }\end{array}$} \\
\hline & & & Mean & SD & Mean & SD & Mean & $\mathrm{SD}$ & Mean & SD \\
\hline \multirow{9}{*}{ Sound } & \multirow{3}{*}{ Bird } & Low & 4.17 & 0.82 & 4.02 & 0.83 & 4.21 & 0.80 & 2.22 & 0.44 \\
\hline & & Medium & 4.27 & 0.68 & 4.15 & 0.79 & 4.30 & 0.70 & 3.24 & 0.34 \\
\hline & & High & 3.41 & 0.96 & 3.34 & 0.89 & 4.27 & 0.67 & 4.13 & 0.26 \\
\hline & \multirow{3}{*}{ Conversation } & Low & 3.40 & 0.82 & 2.96 & 0.92 & 4.23 & 0.73 & 2.30 & 0.55 \\
\hline & & Medium & 2.73 & 0.83 & 2.49 & 0.76 & 4.34 & 0.65 & 3.28 & 0.76 \\
\hline & & High & 2.06 & 0.58 & 1.94 & 0.67 & 4.21 & 0.62 & 4.35 & 0.60 \\
\hline & \multirow{3}{*}{ Traffic } & Low & 2.97 & 0.84 & 2.46 & 0.85 & 4.19 & 0.92 & 2.35 & 0.73 \\
\hline & & Medium & 2.38 & 0.81 & 2.19 & 0.81 & 4.32 & 0.74 & 3.30 & 0.74 \\
\hline & & High & 1.99 & 0.63 & 1.87 & 0.69 & 4.24 & 0.82 & 4.27 & 0.41 \\
\hline \multirow{12}{*}{ Odour } & \multirow{6}{*}{ Osmanthus } & Low & 3.50 & 0.49 & 3.39 & 0.63 & 3.85 & 0.64 & 2.27 & 0.32 \\
\hline & & Medium & 3.67 & 0.59 & 3.67 & 0.59 & 3.87 & 0.60 & 3.07 & 0.64 \\
\hline & & High & 3.18 & 0.70 & 3.20 & 0.83 & 3.98 & 0.65 & 4.00 & 0.45 \\
\hline & & Low & 3.50 & 0.44 & 3.35 & 0.42 & 3.77 & 0.47 & 2.19 & 0.37 \\
\hline & & Medium & 3.65 & 0.88 & 3.59 & 0.89 & 3.78 & 0.66 & 3.00 & 0.68 \\
\hline & & High & 3.33 & 0.70 & 3.28 & 0.70 & 3.88 & 0.53 & 4.00 & 0.50 \\
\hline & \multirow{3}{*}{ Coffee } & Low & 3.58 & 0.77 & 3.39 & 0.82 & 3.92 & 0.59 & 2.31 & 0.25 \\
\hline & & Medium & 3.87 & 0.65 & 3.80 & 0.74 & 4.11 & 0.39 & 3.09 & 0.55 \\
\hline & & High & 3.98 & 1.04 & 3.90 & 0.95 & 4.20 & 1.18 & 4.08 & 0.56 \\
\hline & \multirow{3}{*}{ Bread } & Low & 3.54 & 0.64 & 3.35 & 0.87 & 3.92 & 0.52 & 2.31 & 0.25 \\
\hline & & Medium & 3.72 & 0.55 & 3.61 & 0.68 & 3.96 & 0.44 & 3.07 & 0.34 \\
\hline & & High & 3.78 & 0.69 & 3.70 & 0.85 & 4.10 & 0.92 & 4.15 & 0.48 \\
\hline
\end{tabular}


Fig. 2 shows the mean rating change of sound evaluation indicators relative to the condition of without odour. For acoustic comfort, with the introduction of odour, the mean ratings increased in most cases compared with the condition of without odour (most are positive values). Two plant odours had similar effects on acoustic comfort, while the effects of two food odours were also similar, but the effects of plant and food odours were different. No matter the type of odour, when the concentration was low, the changes of acoustic comfort ratings were mostly less than 0.1 ; when the concentration was medium or high, its influence on acoustic comfort was stronger than that of the low concentration. Specifically, in the case of birdsong, plant odours had a tiny lifting effect on acoustic comfort when the volume was low, while food odours were the opposite. For medium volume, all types of odours reduced acoustic comfort, and with the increase of concentration, the decline was more obvious. For high volume, the plant odours improved the acoustic comfort with the increase of concentration, while the food odours improved the acoustic comfort when the concentration was medium or high and the effect of high concentration was greater. In terms of conversation and traffic sounds, the effects of odour on them were similar. For low and medium volume, the odour improved the acoustic comfort, and the effect of plant odours of medium concentration was the best; however, with the increase of food odour concentration, the effect of improving acoustic comfort got better. Meanwhile, for high volume, the higher the concentration, the more obvious the enhancement effect on acoustic comfort. In sum, the effect of odour on acoustic comfort of traffic sound was the most evident, and that of birdsong was the least. The effect of odour on sound preference was analogous to that on acoustic comfort.

In terms of sound familiarity, with the introduction of odour, the mean ratings increased in most cases compared with the condition of without odour (most are positive values). However, the most changes of rating were less than 0.1 .

As for subjective loudness, the mean ratings declined in most cases compared with the condition of without odour (most are negative values). For all sounds, the higher the concentration of odour, the more evident the drop in subjective loudness. 


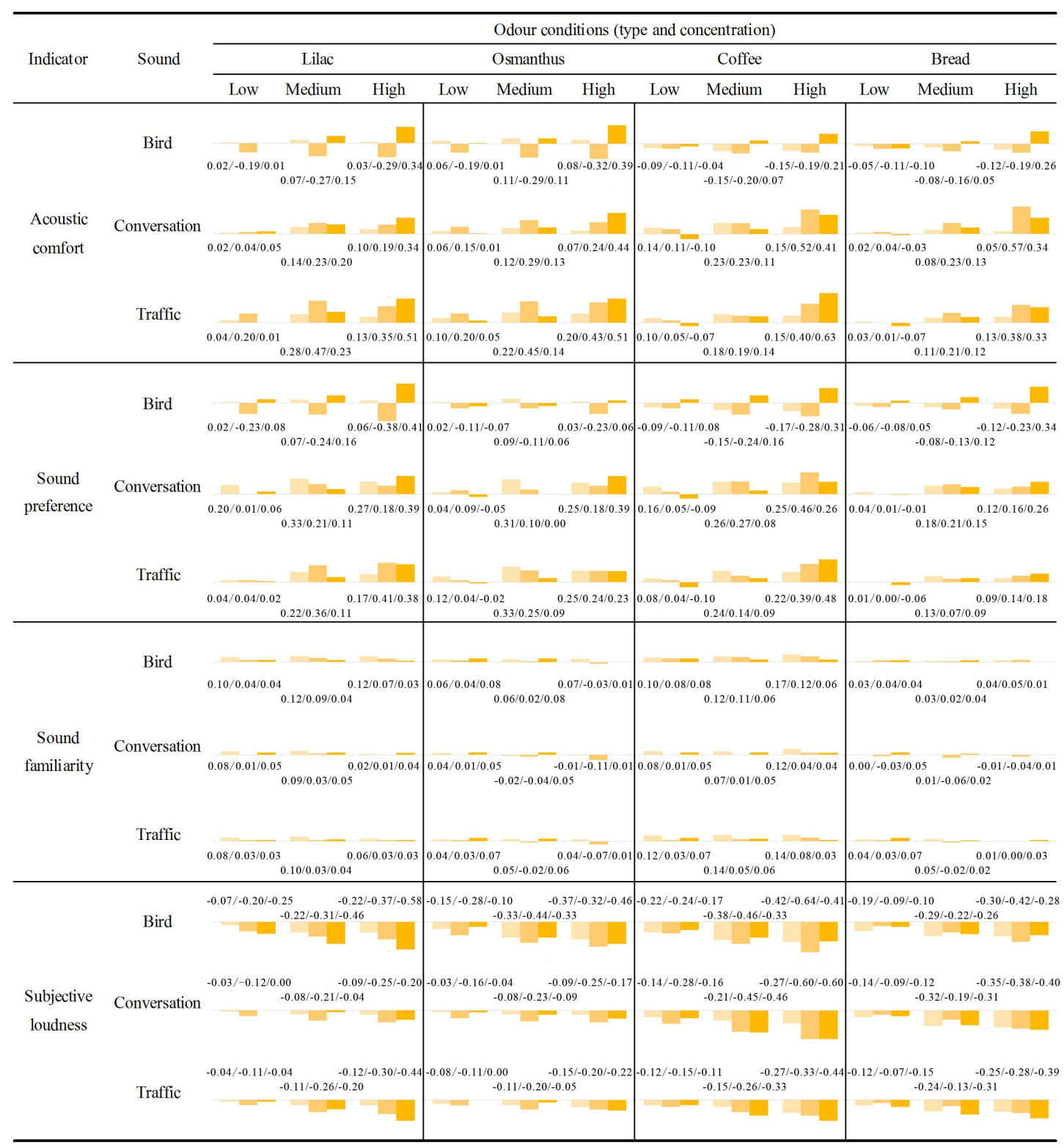

Low volume; Medium volume; $=$ High volume

Fig. 2 Mean rating change of sound evaluation indicators relative to the condition of without odour

\subsubsection{The interaction of sound and odour factors on sound evaluation}

Mixed design analyses of variance (ANOVAs) were run to test the differences in mean ratings between subgroups divided by four main variables. Sound type, volume, odour type, and concentration were taken as independent variables. The significance of the indicators under main effect and interaction are shown in Table 4. Main effects are irrelevant if there are interaction effects. The following sections analyse the effects of both sound and odour factors upon interactions. The interaction of sound and odour factors themselves are not discussed (e.g., the interaction of odour type and concentration, or sound type and volume are out of consideration). The results showed that all mean ratings for the indicators were affected by the interaction of sound and odour factors except for sound and odour familiarities. 
Table 4 The significances of the indicators under main effect and interaction

\begin{tabular}{|c|c|c|c|c|c|c|c|c|c|c|}
\hline Source & $\begin{array}{l}\text { Acoustic } \\
\text { comfort }\end{array}$ & $\begin{array}{c}\text { Sound } \\
\text { preference }\end{array}$ & $\begin{array}{c}\text { Sound } \\
\text { familiarity }\end{array}$ & $\begin{array}{l}\text { Subjective } \\
\text { loudness }\end{array}$ & $\begin{array}{l}\text { Olfactory } \\
\text { comfort }\end{array}$ & $\begin{array}{c}\text { Odour } \\
\text { preference }\end{array}$ & $\begin{array}{l}\text { Odour } \\
\text { familiarity }\end{array}$ & $\begin{array}{l}\text { Subjective } \\
\text { intensity }\end{array}$ & $\begin{array}{l}\text { Overall } \\
\text { comfort }\end{array}$ & Congruency \\
\hline Odour type & .175 & .122 & .093 & .000 & .000 & .000 & .000 & .000 & .012 & .001 \\
\hline Sound type & .000 & .000 & .299 & .000 & .000 & .000 & .505 & .000 & .000 & .000 \\
\hline Volume & .000 & .000 & .001 & .000 & .000 & .000 & .751 & .000 & .000 & .000 \\
\hline Odour type * Sound type & .423 & .564 & 1.000 & .000 & .000 & .000 & .259 & .000 & .088 & .000 \\
\hline Odour type ${ }^{*}$ Volume & .673 & .124 & .873 & .001 & .275 & .930 & 1.000 & .102 & .247 & .044 \\
\hline Concentration $*$ Sound type & .013 & .010 & .997 & .702 & .580 & .609 & .984 & .848 & .000 & .031 \\
\hline Concentration * Volume & .000 & .000 & .989 & .035 & .979 & .838 & 1.000 & .519 & .000 & .044 \\
\hline $\begin{array}{c}\text { Odour type } * \text { Concentration } \\
* \text { Volume }\end{array}$ & .811 & .983 & 1.000 & .978 & 1.000 & 1.000 & 1.000 & .959 & .846 & .507 \\
\hline $\begin{array}{c}\text { Odour type } * \text { Sound type } * \\
\text { Volume }\end{array}$ & .260 & .186 & 1.000 & .051 & .541 & .344 & 1.000 & .456 & .059 & .052 \\
\hline $\begin{array}{c}\text { Concentration } * \text { Sound type } \\
* \text { Volume }\end{array}$ & .400 & .711 & 1.000 & .850 & .999 & .995 & .998 & .964 & .057 & .991 \\
\hline $\begin{array}{l}\text { Odour type } * \text { Concentration } \\
* \text { Sound type } * \text { Volume }\end{array}$ & 1.000 & 1.000 & 1.000 & 1.000 & 1.000 & 1.000 & 1.000 & .996 & .890 & 999 \\
\hline
\end{tabular}

Note: ${ }^{\text {a }}$ Significance (at 0.05 ) is in bold ${ }^{\mathrm{b}}$ Contrasts adjusted for multiple comparisons with Scheffe adjustment.

A one-way ANOVA was run to test the differences in mean ratings between subgroups divided by the variables that interacted. After removing the factors without statistical significance, the model was simplified. The mean ratings of sound evaluation indicators are shown in Fig. 3. For acoustic comfort, sound type and volume interacted with concentration The corresponding mean ratings of acoustic comfort under their interactions are shown in Fig. 3a and b. The results of multiple comparison analysis under the interaction of sound types and concentration showed that concentration did not affect acoustic comfort for birdsong $(p=0.496>0.05)$. For conversation and traffic sounds, the acoustic comfort rose gradually with the increase of concentration. The interaction of volume and concentration showed that concentration did not affect acoustic comfort for low-volume sound; for medium volume, acoustic comfort in the presence of odour was higher than that in the case of no odour $(p=0.000<0.01)$, and the higher the concentration, the higher the acoustic comfort. As for high-volume sounds, odours of high concentration had significant effect on the improvement of its acoustic comfort.

In terms of sound preference, there was interaction between sound type, volume, and concentration, which was similar to acoustic comfort. The mean ratings of sound preference under the interactions are shown as Fig. $3 \mathrm{c}$ and $\mathrm{d}$. The interaction between sound type and concentration showed that concentration did not affect sound preference for birdsong $(\mathrm{p}=0.886>0.05)$. For conversation and traffic sounds, with the increase of odour concentration, sound preference 
gradually improved. The interaction between volume and concentration showed that concentration did not affect sound preference of low- and medium-volume sounds; for high-volume sound, high-concentration odours had a significant effect on the improvement of its sound preference.

As for subjective loudness, there were interactions between sound type and odour type, volume and odour type, and volume and concentration. The mean ratings of subjective loudness under their interactions are shown in Fig. 3e, f, and 3g. The interaction between sound and odour types showed that odour type did not affect subjective loudness of bird and traffic sounds, but there was a significant difference between with and without odours $(\mathrm{p}=0.000<0.01)$. For conversation sound, there was a significant difference between plant and food odours $(\mathrm{p}=0.000<$ 0.01 ), and the subjective loudness declined more by food odour. The interaction between volume and odour type showed that for low- and high-volume sounds, the decline of subjective loudness under the effect of food odours was greater than that of plant odours. For medium-volume sounds, the effect of coffee odour on the decline of subjective loudness was the greatest, while the effect of bread odour was the opposite. The interaction between volume and concentration showed that no matter what the volume was, the higher the concentration, the greater the decline in subjective loudness.
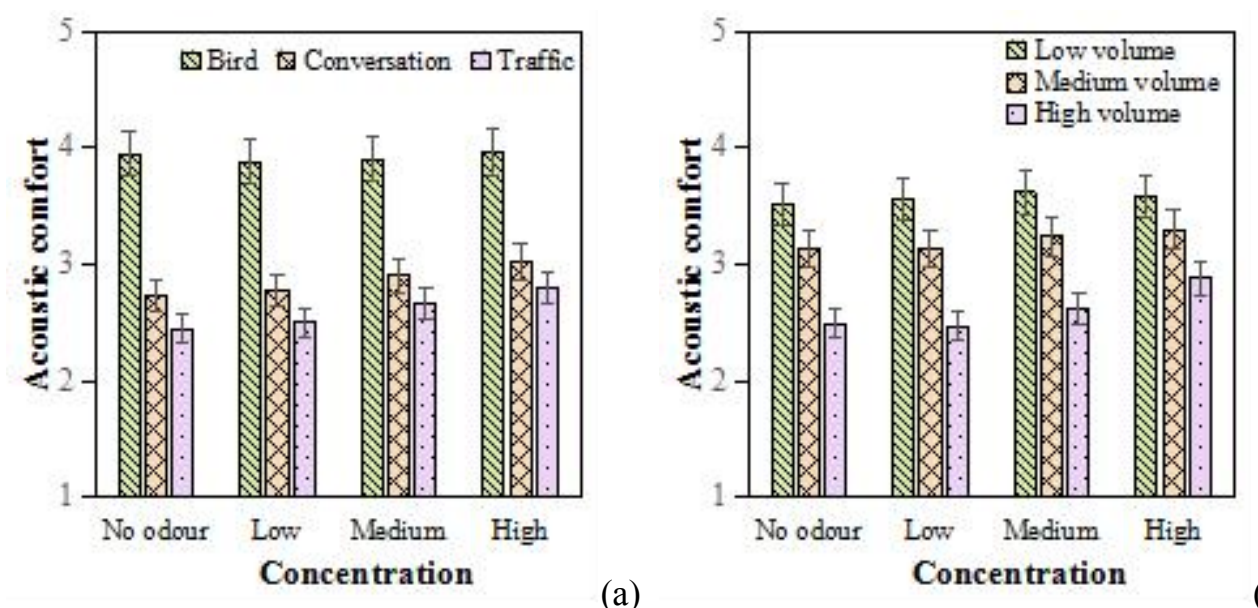

(b)
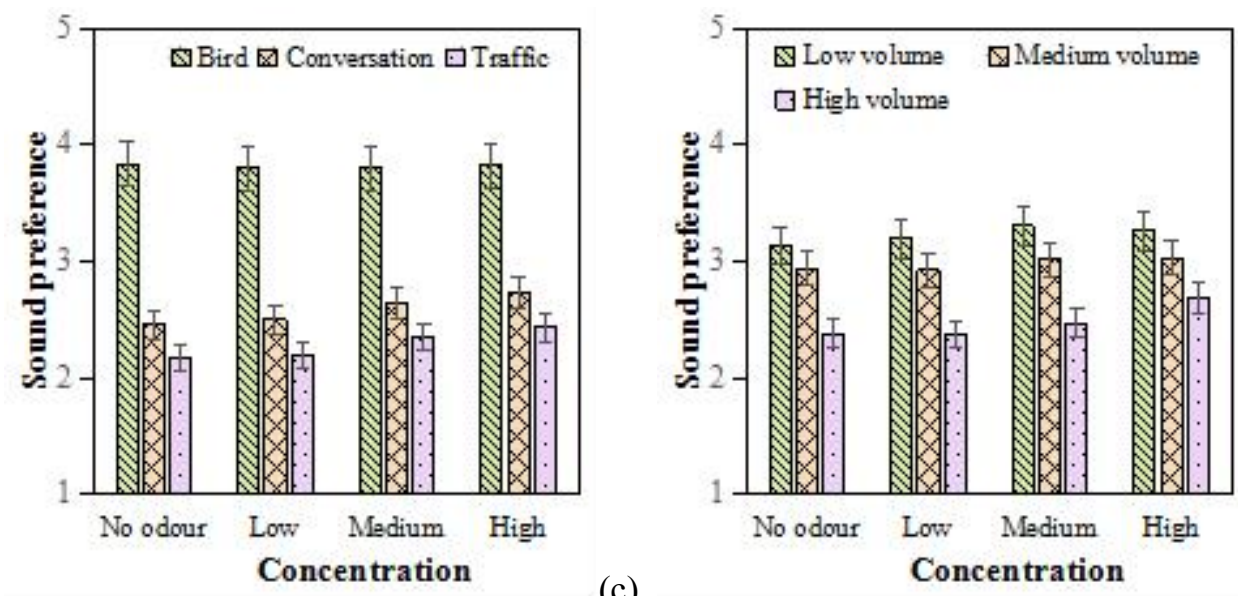

(c) 

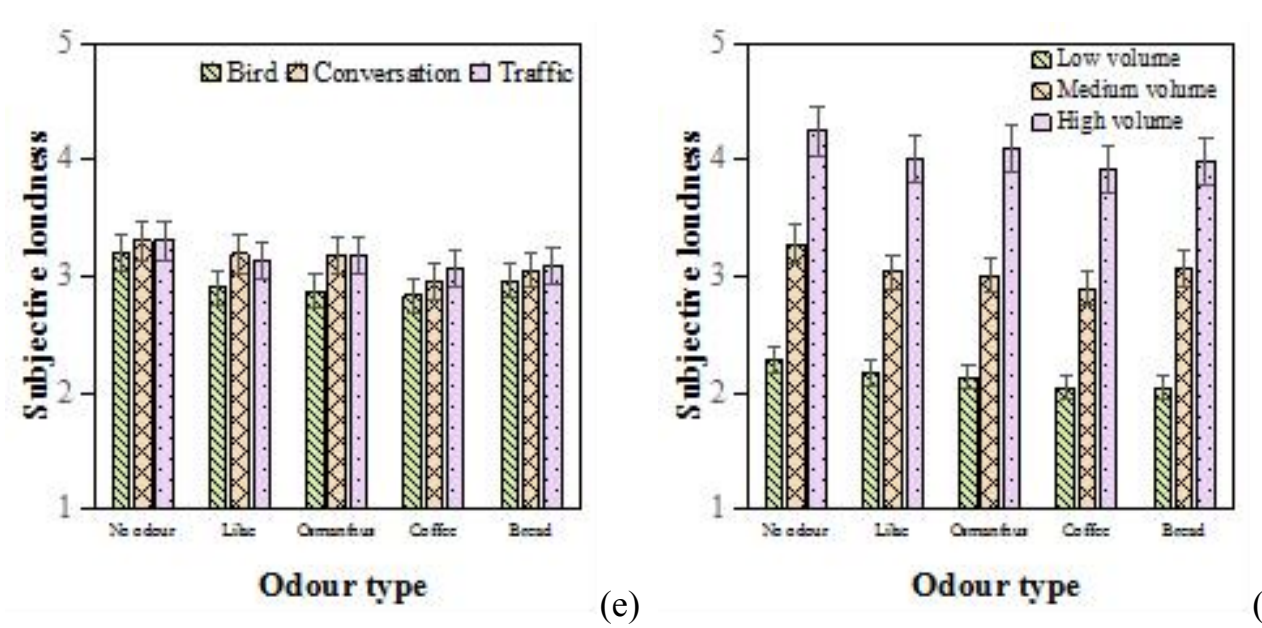

(e)

(f)

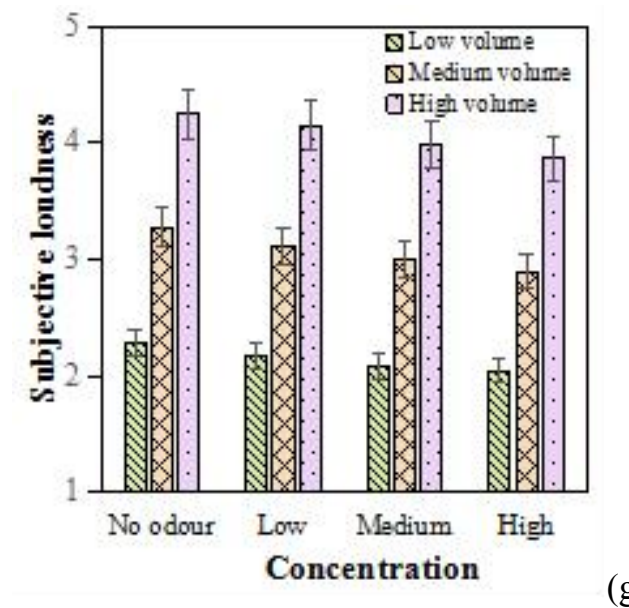

Fig. 3 The mean ratings of sound evaluation indicators under the effect of sound and odour factors

\subsection{Odour evaluation}

\subsubsection{The effect of sound stimuli}

Odour evaluations without sound stimuli are shown in Table 3. In terms of olfactory comfort, a comparison shows that the mean ratings of food odours were higher than those of plant odours, and the olfactory comfort of plant odours were the highest when the concentration was medium, and lowest when the concentration was high. The olfactory comfort of food odours rose with increased concentration, which was consistent with a previous study [40]. Odour preference change was similar to olfactory comfort. The mean ratings for odour familiarity were high and rose with increased concentration. For subjective intensity, whatever the odours, their ratings were very close when the concentration was low, medium or high. This is consistent with the aim that taking the concentrations which corresponded to the subjective evaluation of light, moderate, and strong as the intensity for odour stimuli in Section 2.2.

Fig. 4 shows the mean rating change of odour evaluation indicators relative to the condition of without sound. For olfactory comfort, with the introduction of sound, the mean ratings decreased in most cases compared with the condition of without sound (most are negative values). Conversation and traffic sounds had similar effects on olfactory comfort. In terms of birdsong, high volume resulted in the greatest reduction in ratings, while conversation and traffic sounds reduced the ratings gradually with the increase of volume. Specifically, for plant odours at low and 
high concentrations, birdsong of low and medium volumes had a slight effect on the improvement of olfactory comfort, while high-volume birdsong reduced the ratings. Conversation and traffic sound reduced the olfactory comfort, and the decline was more obvious with increased volume. For plant odours at medium concentrations, all sounds reduced olfactory comfort, and the decline was more obvious when the volume rose. For food odours, the introduction of sound reduced olfactory comfort no matter the concentration, and the decline was more obvious with increased volume. For odour preference, the effect of sound was analogous to that on olfactory comfort.

In terms of odour familiarity, with the introduction of sound, the most change of mean ratings were less than 0.1 . As for subjective intensity, the mean ratings decreased in most cases compared with the condition of without sound (most are negative values). Also, for all odours, the higher the volume, the more evident the drop of subjective intensity. 


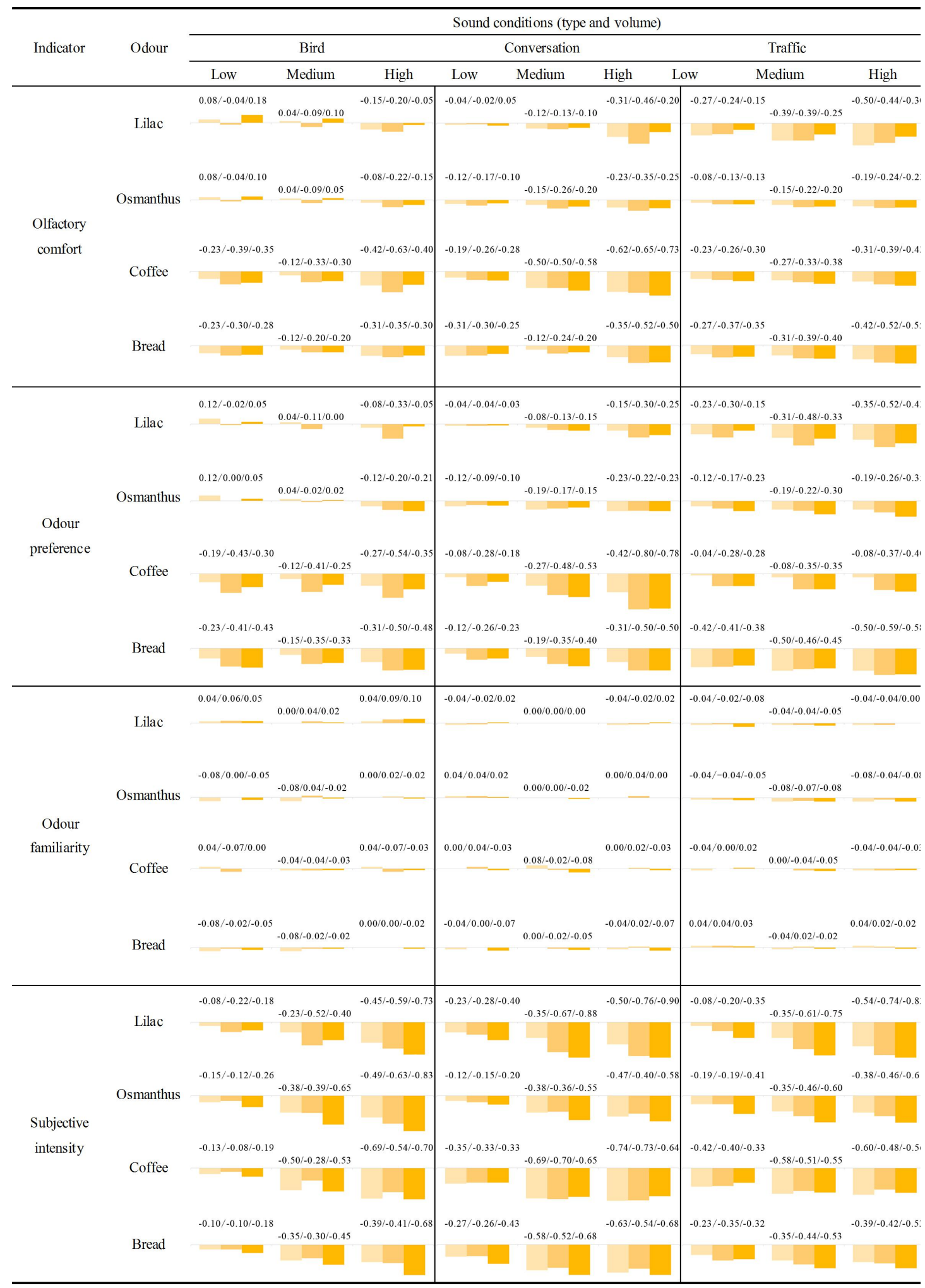

Low concentration; $\approx$ Medium concentration; $\approx$ High concentration

Fig. 4 Mean rating change of odour evaluation indicators relative to the condition of without sound

\subsubsection{The interaction of sound and odour factors on odour evaluation}

The mean ratings of odour evaluation indicators are shown in Fig. 5. Volume had a 
significant effect on all indicators except for odour familiarity, and there was also interaction between odour type and sound type, as shown in Table 4. Mean ratings for olfactory comfort at different volumes are shown in Fig. 5a. There was no significant difference between the effects of low and medium volumes on olfactory comfort $(p=0.435>0.05)$, and the higher the volume, the more obvious the decline of olfactory comfort. The mean ratings of olfactory comfort under the interaction of odour type and sound type are shown in Fig. 5b. For lilac and bread odours, there was no significant difference between the effect of traffic and conversation sounds. The decline of olfactory comfort associated with lilac was the greatest. For osmanthus and coffee odours, there was no significant difference between the effects of three sounds in reducing olfactory comfort.

The mean ratings for odour preference at different volumes are shown in Fig. 5c. There was no significant difference between the effects of low and medium volume on odour preference $(p=0.125>0.05)$; odour preference decreased with the increase of volume. The mean ratings of odour preference under the interaction of odour type and sound type are shown in Fig. 5d. The effect of traffic sound on the decline of lilac odour preference was the most obvious $(p=0.000<$ $0.01)$. For osmanthus odour, there was no significant difference between the effects of traffic or of conversation sounds on its preference $(p=0.738>0.05)$, and their effects were greater than birdsong. For food odours, there was no significant difference among the three types of sounds for the decline of odour preference.

The mean ratings for subjective intensity at different volumes are shown in Fig. 5e. There was a significant difference in the influence of three volumes on subjective intensity ( $p=0.000<$ 0.01 ); the ratings decreased as volume increased. The mean ratings of subjective intensity under the interaction of odour type and sound type are shown in Fig. 5f. For all odours, there was no significant difference in the effects of three sounds on subjective intensity but there was significant difference with the no-sound condition.
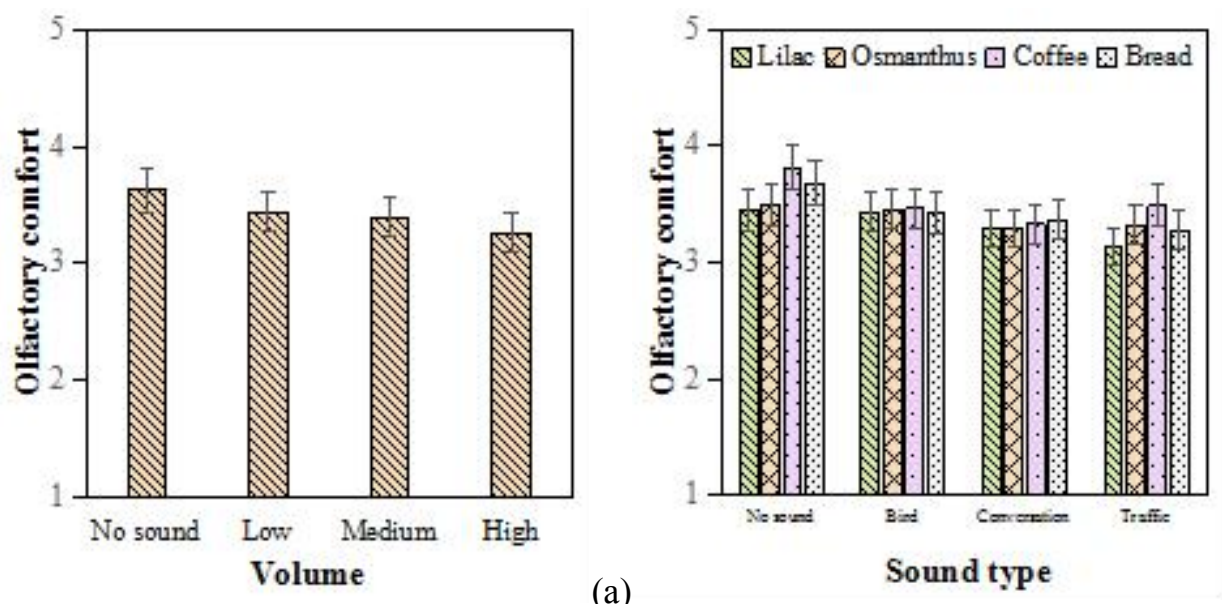

(a) 

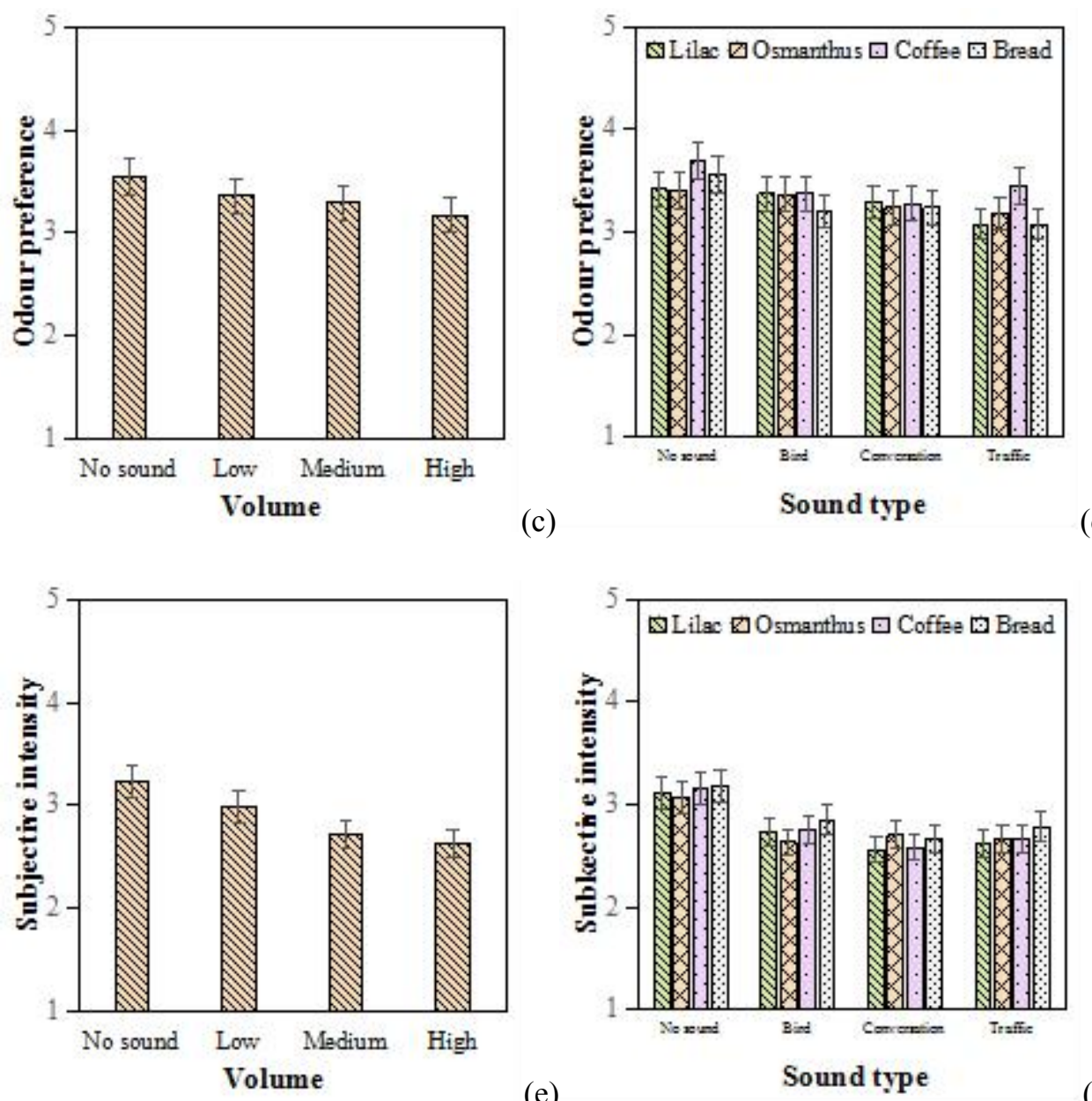

(e)

Fig. 5 The mean ratings of odour evaluation indicators under the effect of sound and odour factors

\subsection{Overall evaluation}

\subsubsection{The effect of sound and odour stimuli}

Table 5 shows the overall evaluation under the effect of sound and odour stimuli. The introduction of plant odour and the concentration increase caused the overall comfort rating between plant odours and low-volume birdsong gradually to decline. The overall comfort between plant odours and high-volume birdsong was the opposite, and the overall comfort between plant odours and medium-volume birdsong increased at first and then decreased. With the introduction of coffee odour and the increase of concentration, the level of overall comfort between the odour and birdsong with different volumes gradually increased; however, an increase of concentration of bread odour caused overall comfort level to decline. For the conversation and traffic sounds together with the plant odour, the overall comfort of low and medium volumes reached the highest value when the concentration was moderate, and the overall comfort of high volume rose with increased concentration. With the introduction of food odour and the increase of concentration, the overall comfort gradually increased.

For the congruency between sound and odour, with the introduction of plant odour and the increase of concentration, the congruency between plant odour and low-volume birdsong 
gradually decreased. In the presence of medium volume, the congruency increased at first and then decreased, and the congruency between high-volume birdsong and lilac odour rose gradually, while it had no obvious change with osmanthus odour. With the introduction of coffee odour and the increase of concentration, the congruency between odour and birdsong increased gradually; the change of congruency between bread odour and low- or medium-volume birdsong was opposite to that of coffee odour, and the congruency between bread odour and high-volume birdsong was the highest at medium concentration and the lowest at high concentration. For conversation and traffic sounds, with the introduction of plant odour, the congruency between plant odour and low-volume or medium-volume sound was the highest when the concentration was medium. The congruency between plant odour and high-volume sound rose with the increase of concentration, and with the introduction of food odour and increased concentration, the congruency gradually increased.

Table 5 Overall evaluation under the effect of sound and odour stimuli

\begin{tabular}{|c|c|c|c|c|c|c|c|c|c|c|c|c|c|c|}
\hline \multirow{3}{*}{ Indicator } & \multirow{3}{*}{ Sound } & \multirow{3}{*}{ Volume } & \multicolumn{12}{|c|}{ Odour conditions (types and concentration) } \\
\hline & & & \multicolumn{3}{|c|}{ Lilac } & \multicolumn{3}{|c|}{ Osmanthus } & \multicolumn{3}{|c|}{ Coffee } & \multicolumn{3}{|c|}{ Bread } \\
\hline & & & Low & Medium & High & Low & Medium & High & Low & Medium & High & Low & Medium & High \\
\hline \multirow{9}{*}{$\begin{array}{l}\text { Overall } \\
\text { comfort }\end{array}$} & \multirow{6}{*}{ Conversation } & Low & 3.92 & 3.89 & 3.80 & 4.08 & 4.02 & 3.90 & 3.85 & 3.89 & 4.08 & 3.92 & 3.74 & 3.70 \\
\hline & & Medium & 3.73 & 3.83 & 3.70 & 3.77 & 3.85 & 3.63 & 3.69 & 3.70 & 3.73 & 3.77 & 3.70 & 3.63 \\
\hline & & High & 3.46 & 3.54 & 3.65 & 3.31 & 3.41 & 3.38 & 3.19 & 3.28 & 3.63 & 3.27 & 3.35 & 3.23 \\
\hline & & Low & 3.39 & 3.57 & 3.25 & 3.19 & 3.33 & 3.13 & 3.42 & 3.48 & 3.60 & 3.08 & 3.15 & 3.38 \\
\hline & & Medium & 2.62 & 3.04 & 2.98 & 2.92 & 3.09 & 3.00 & 2.89 & 3.00 & 3.43 & 2.81 & 2.96 & 3.23 \\
\hline & & High & 1.69 & 2.07 & 2.48 & 2.04 & 2.17 & 2.38 & 1.96 & 2.13 & 2.28 & 1.85 & 1.94 & 2.40 \\
\hline & \multirow{3}{*}{ Traffic } & Low & 3.04 & 3.22 & 2.95 & 3.19 & 3.26 & 3.08 & 2.96 & 3.02 & 3.38 & 3.04 & 3.09 & 3.23 \\
\hline & & Medium & 2.65 & 2.80 & 2.73 & 2.62 & 2.98 & 2.93 & 2.39 & 2.70 & 2.98 & 2.62 & 2.70 & 2.78 \\
\hline & & High & 1.92 & 2.30 & 2.58 & 1.77 & 2.26 & 2.55 & 1.89 & 2.00 & 2.78 & 1.89 & 2.00 & 2.63 \\
\hline \multirow{9}{*}{ Congruency } & \multirow{6}{*}{ Conversation } & Low & 3.54 & 3.50 & 3.23 & 3.35 & 3.30 & 3.28 & 3.19 & 3.28 & 3.45 & 3.46 & 3.39 & 3.33 \\
\hline & & Medium & 3.35 & 3.39 & 3.15 & 3.12 & 3.15 & 3.08 & 3.00 & 3.07 & 3.18 & 3.31 & 3.24 & 3.18 \\
\hline & & High & 2.81 & 3.02 & 3.13 & 2.92 & 2.98 & 2.95 & 2.65 & 2.74 & 3.08 & 2.92 & 2.98 & 2.88 \\
\hline & & Low & 3.27 & 3.44 & 3.30 & 3.23 & 3.26 & 3.15 & 3.50 & 3.59 & 3.75 & 3.15 & 3.28 & 3.45 \\
\hline & & Medium & 2.96 & 3.13 & 3.10 & 2.77 & 3.00 & 2.90 & 2.81 & 3.04 & 3.53 & 3.04 & 3.13 & 3.20 \\
\hline & & High & 2.39 & 2.57 & 2.85 & 2.35 & 2.54 & 2.78 & 2.27 & 2.57 & 2.75 & 2.92 & 2.94 & 2.98 \\
\hline & \multirow{3}{*}{ Traffic } & Low & 2.46 & 2.65 & 2.43 & 2.81 & 2.87 & 2.78 & 2.96 & 3.09 & 3.23 & 2.73 & 2.87 & 2.93 \\
\hline & & Medium & 2.08 & 2.35 & 2.25 & 2.50 & 2.76 & 2.60 & 2.54 & 2.65 & 2.90 & 2.31 & 2.44 & 2.60 \\
\hline & & High & 1.85 & 2.09 & 2.23 & 2.35 & 2.46 & 2.58 & 2.39 & 2.44 & 2.50 & 1.81 & 1.96 & 2.18 \\
\hline
\end{tabular}

\subsubsection{The interaction of sound and odour factors on overall evaluation}

The mean ratings of overall evaluation indicators are shown in Fig. 6. Odour type had a significant effect on the overall comfort rating, and there was also an interaction between sound type and concentration, as shown in Table 4. The mean ratings at different odour types are shown in Fig. 6a. The effect of bread and lilac odours on overall comfort was the same $(\mathrm{p}=0.056>0.05)$. There was also no significant difference between the effect of the lilac, osmanthus, and coffee odours $(\mathrm{p}=0.957>0.05)$. Their effects on overall comfort were greater than that of bread odour. 
The mean ratings of overall comfort under the interaction of sound type and concentration are shown in Fig. 6b. Concentrations did not affect the overall comfort for birdsong ( $\mathrm{p}=0.909>0.05$ ). For conversation and traffic sounds, the overall comfort rose with the increased concentration. The mean ratings of overall comfort under the interaction of volume and concentration are shown in Fig. 6c. Concentration did not affect overall comfort for low-volume sound $(\mathrm{p}=0.605>0.05)$. For medium- and high-volume sounds, the overall comfort also gradually rose with increased concentration.

In terms of the congruency between sound and odour, its mean ratings under the interaction of sound type and odour type are shown in Fig. 6d. Odour type did not affect the congruency for birdsong $(\mathrm{p}=0.094>0.05)$. The congruency between conversation sound and food odour was higher than that of plant odour. For traffic sound, the effects of the odours of osmanthus and coffee were the same $(p=0.265>0.05)$, and the congruency between traffic sound and osmanthus or coffee odour was higher than that of bread or lilac odour. The mean ratings of congruency under the interaction of volume and odour type are shown in Fig. 6e. Coffee odour had a greater effect on the improvement of congruency for low-volume sound; for medium- and high-volume sounds, odour type did not affect the congruency. The mean ratings of congruency under the interaction of sound type and concentration are shown in Fig. 6f. Concentration did not affect the congruency for birdsong $(\mathrm{p}=0.808>0.05$ ). For conversation and traffic sounds, the rating of congruency gradually rose with increased concentration. The mean ratings of congruency under the interaction of volume and concentration are shown in Fig. 6g. Concentration did not affect the congruency for low volume; for medium and high volume, the congruency of medium or high concentrations was higher than that of low concentration.
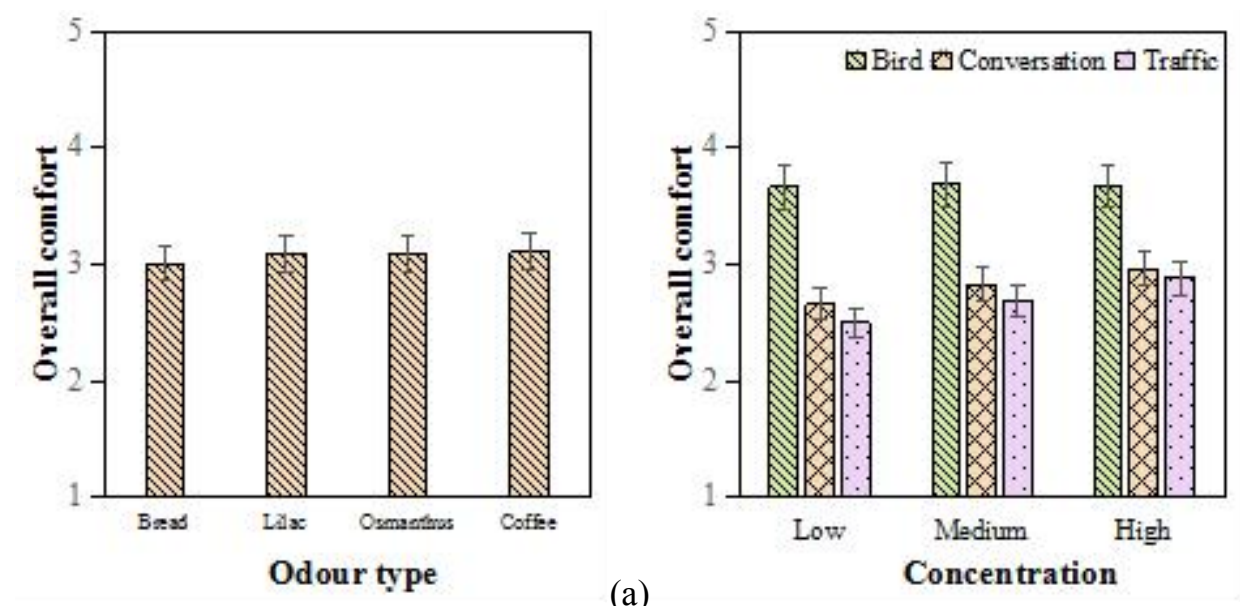

(b) 

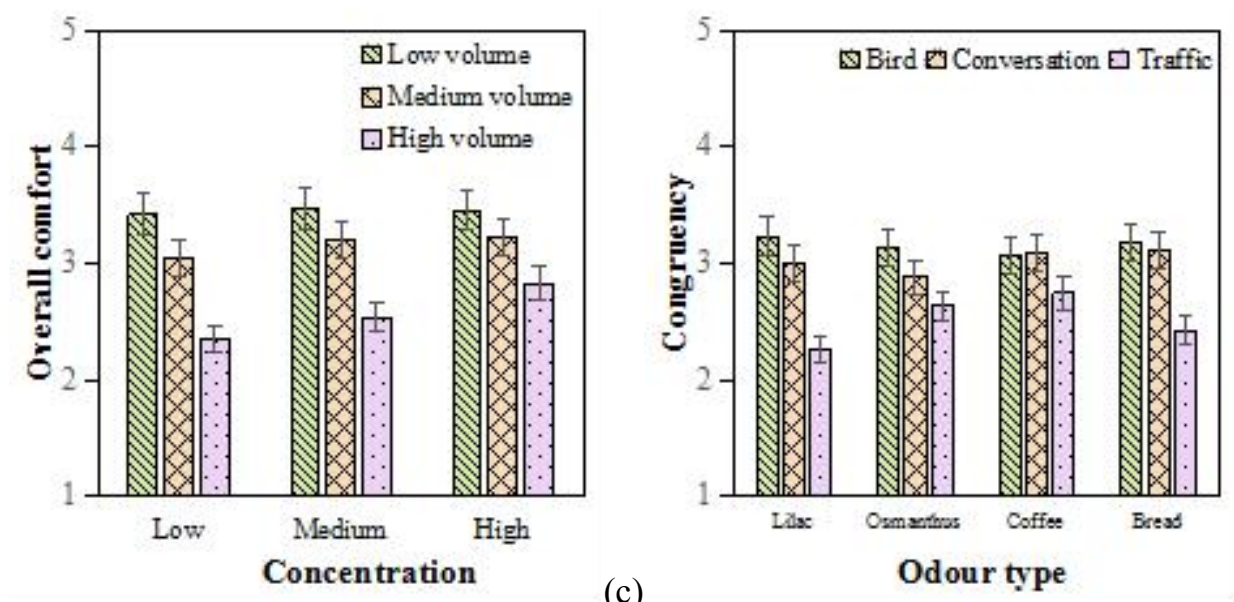

(c)
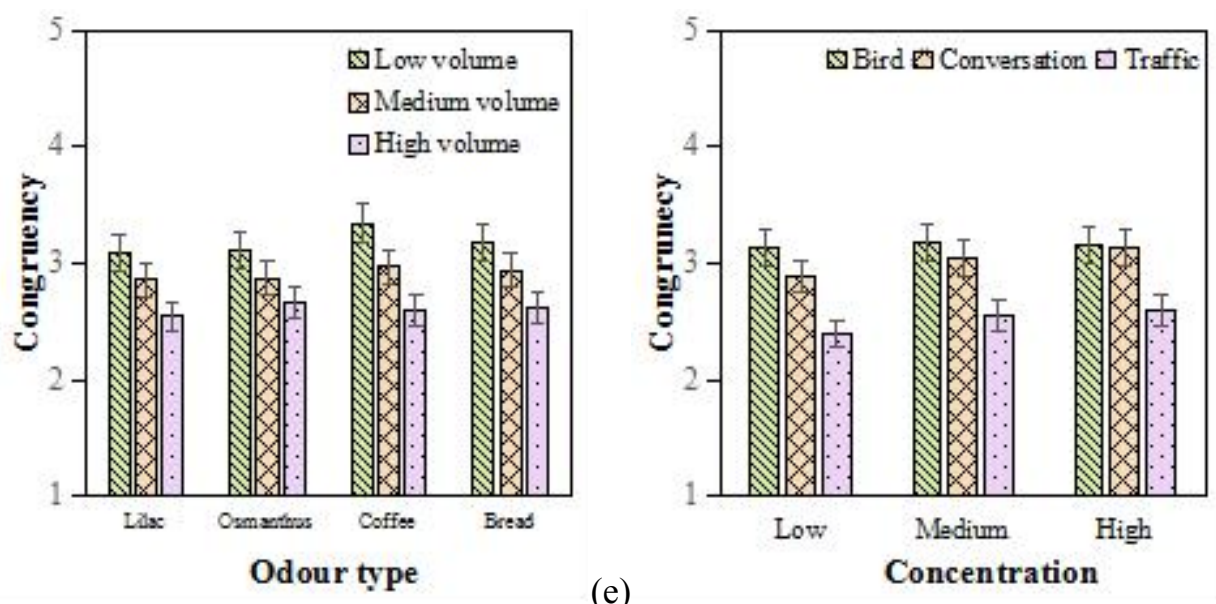

(e)

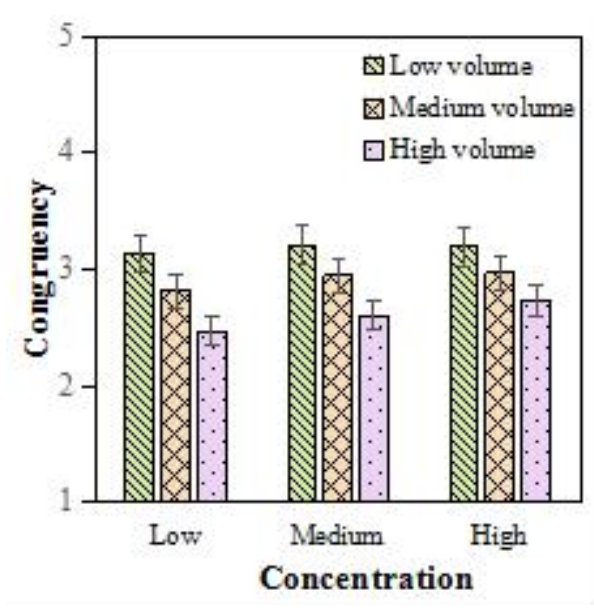

(g)

Fig. 6 The mean ratings of overall evaluation indicators under the effect of sound and odour factors 


\section{Discussion}

\subsection{Similarities and differences in the evaluations of sound and odour under the effect of each other}

Sections 3.1 and 3.2 describe the similarities and differences in the evaluation of sound and odour evaluations under their mutual effects. Spearman correlations were run to test the correlations between evaluation indicators. The results showed that there was a similar trend between sensory comfort and preference, with a correlation coefficient between acoustic comfort and sound preference of 0.88 . The correlation coefficient between olfactory comfort and odour preference was 0.829 . Previous studies have shown that people feel more comfortable in a sound or smell environment that they favour $[41,42]$. The results of this study showed that whether the stimulus is single-sensory or multi-sensory, this trend still exists. Moreover, the sensory familiarity was not affected by other sensory factors. It may be that because the familiarities of the sensory samples selected in this study are high, they could hardly be influenced by each other. For subjective loudness and subjective intensity, their perceptions declined with the increase of the stimulation intensity of each other. Previous studies have found such mutual masking between vision and audition [5,34]. The results of this study showed that this relationship also exists between audition and olfaction.

Regarding the differences in the evaluation of sound and of odour, the introduction of odour improved the evaluation of acoustic comfort and sound preference in most cases. In the case of olfactory comfort and odour preference, however, the introduction of sound reduced their evaluations the most. A possible reason is that, except for birdsong, conversation and traffic sounds are negatively perceived sounds, while the samples of food and plant odours were neutral or positive in this study. Thus, it is evident that positive sensory stimulation can improve the evaluation of another sense, while negative sensory stimulation can deteriorate the evaluation of another sense $[43,44]$.

\subsection{The relationship between the sensory evaluation and the overall evaluation}

The correlation coefficient between acoustic comfort and overall comfort was 0.790 , the correlation coefficient between olfactory comfort and overall comfort was 0.407. These correlations between the evaluations of sound and odour and overall comfort showed that the effect of acoustic comfort on overall comfort was greater. The correlation between the evaluations of sound and odour and the congruency was expressed by a correlation coefficient between acoustic comfort and the congruency, which was 0.456 . The correlation coefficient between olfactory comfort and the congruency was 0.523 . This indicated that the effects of acoustic comfort and olfactory comfort on the congruency were almost the same. Previous studies have demonstrated that vision plays a leading role among all the senses, and audition takes a major supporting role $[45,46]$. This study showed that audition plays a dominant role in the perception of sound and odour interaction. However, when it comes to the congruency of two stimuli, the effects of sound and odour are balanced.

For overall evaluation, the correlation coefficient between overall comfort and congruency was 0.539 , that is, the higher the congruency, the more comfortable people feel. The result also suggested that even if a single odour or sound is pleasant, a sensory stimulus is comfortable only 
when it is congruent with the surroundings in which it is perceived; otherwise, it can still make people feel uncomfortable. In fact, by contrast, even annoying odours and noises do not generate excess revulsion when they are perceived as being in harmony with the environment [47].

\subsection{Implications for future research}

The current study focused specifically on perceptual responses to the combination of sensory stimuli to inform broad recommendations for sensory environment and to identify future research needed. This study could be expanded in a number of directions:

- Even if the simulation variable control is accurate in the laboratory, it cannot replace the real feeling of the actual environment on site and in real time. The results of laboratory studies should be verified in the field [48].

- The three sound samples selected in this study are dominated by different sound sources, and the odour samples are evaluated as neutral and positive. More types of sounds and odours can be added in future research. Negative odours could also be chosen for multisensory research. The familiarity of sounds and odours selected in this study was high. Future research can choose moderate or unfamiliar sensory stimulation.

- The effect of user's characteristics on the perception of aural-olfactory simulations should be taken into consideration ( such as gender, age, cultural difference, professional background, etc [49-51]).

- Because of the dominant role of vision in the five senses, its influence cannot be underestimated. Future experiments can add visual factors as variables to study the interaction of vision, audition and olfaction.

\subsection{Implications for practice}

The results of this study are useful to urban spatial layout design and landscape plant selection. When the sound or smell environment in a particular area is unsatisfactory, the introduction of another positive sensory stimulus, such as setting up a bakery or growing aromatic plants next to a street with too much traffic noise, can improve the situation. The masking effect between audition and olfaction shows that when certain sensory stimuli in the area are too strong, causing people's discomfort, properly increasing the intensity of another sensory stimulus can distract people's attention and thus improve their comfort. When the sound and smell environment in the area both need to be improved, giving priority to improving the sound environment may be more helpful to improve the overall comfort.

\section{Conclusions}

This study investigated the interaction of sound and odour in urban environments. A laboratory experiment was carried out where participants evaluated sound evaluation indicators (acoustic comfort, sound preference, sound familiarity, subjective loudness), odour evaluation 
indicators (olfactory comfort, odour preference, odour familiarity, subjective intensity), and overall evaluation indicators (overall comfort and congruency).

The influence of odour on sound evaluation showed that the presence of odour has little effect on the evaluations of birdsong and low-volume sound, whereas for other types or volumes of sounds, the higher the concentration, the more positive the evaluation.

The influence of sound on odour evaluation showed that an increase of volume can turn odour evaluations negative; in such cases, traffic and conversation sounds have the greatest effects.

For overall evaluation, in the presence of birdsong and low-volume sound, overall comfort and congruency are unaffected by odour; for other combinations of sound and odour, with the increase of concentration, the overall evaluation gradually improves.

The similarities and differences in the evaluations of sound and odour also showed that there is an analogous trend of sensory comfort and preference because of their high correlation coefficient. A positive sensory stimulus can improve the evaluation of perception through other senses, while a negative sensory stimulus has the opposite effect. There is a masking effect between audition and olfaction, which is reflected in the fact that the stronger one stimulus is, the weaker the perceptual intensity of the other is. However, sensory familiarity is not changed by other senses. Moreover, the relationship between sensory evaluation and overall evaluation showed that, for overall comfort, the effect of sound is stronger than odour, while for congruency, the effects of sound and odour are almost equal.

\section{Funding}

This work was supported by the National Natural Science Foundation of China [grant number 51778169]. 


\section{References}

[1] A. Krishna, Customer Sense: How the 5 Senses Influence Buying Behavior, 2013, Springer https://doi.org/10.1093/chemse/20.5.479.

[2] J.A. Smith, Aristotle: 'On the Soul', 1931.

[3] M.L. Ye, Five Senses Applied in Landscape Design, Doctoral dissertation2009, Tianjin University.

[4] C. Spence, The handbook of multisensory processes, In: The Handbook of Multisensory Processes, 2004, MIT Press.

[5] M.F. Southworth, The Sonic Environment of Cities, Doctoral dissertation1967, Massachusetts Institute of Technology.

[6] J. Carles, F. Bernáldez and J.D. Lucio, Audio - visual interactions and soundscape preferences, Landsc. Res. 17 (2), 1992, 52-56 https://doi.org/10.1080/01426399208706361.

[7] S. Viollon, Two examples of audio-visual interactions in an urban context, Acta Acustica 89, 2003, S58.

[8] F. D'Alessandro, L. Evangelisti, C. Guattari, G. Grazieschi and F. Orsini, Influence of visual aspects and other features on the soundscape assessment of a university external area, Build. Acoust. 25 (3), 2018, 199-217 https://doi.org/10.1177/1351010X18778759.

[9] L. Maffei, T. Iachini, M. Masullo, F. Aletta, F. Sorrentino, V.P. Senese and F. Ruotolo, The effects of vision-related aspects on noise perception of wind turbines in quiet areas, Int. J. Environ. Res. Publ. Health 10 (5), 2013, 1681-1697, https://doi.org/10.3390/ijerph10051681.

[10] X. Ren and J. Kang, Effects of the visual landscape factors of an ecological waterscape on acoustic comfort, Appl. Acoust. 96, 2015, 171-179 https://doi.org/10.1016/j.apacoust.2015.03.007.

[11] J. Liu, J. Kang, H. Behm and T. Luo, Effects of landscape on soundscape perception: soundwalks in city parks, Landsc. Urban Plann. 123, 2014, 30-40 https://doi.org/10.1016/j.landurbplan.2013.12.003.

[12] J.L. Carles, I.L. Barrio and J.V. de Lucio, Sound influence on landscape values, Landsc. Urban Plann. 43 (4), 1999, 191-200 https://doi.org/10.1016/S0169-2046(98)00112-1.

[13] B.L. Mace, P.A. Bell, R.J. Loomis and G.E. Haas, Source Attribution of Helicopter Noise in Pristine National Park Landscapes, 2003, American Academy for Park and Recreation Administration.

[14] M. Lindquist, E. Lange and J. Kang, From 3D landscape visualization to environmental simulation: the contribution of sound to the perception of virtual environments, Landsc. Urban Plann. 148, 2016, 216-231 https://doi.org/10.1016/j.landurbplan.2015.12.017.

[15] C.M. Van Reekum, H. vann de Berg and N.H. Frijda, Cross-modal preference acquisition: evaluative conditioning of pictures by affective olfactory and auditory cues, Cognit. Emot. 13 (6), 1999, 831-836 https://doi.org/10.1080/026999399379104. 
[16] H.Q. Dinh, N. Walker, L.F. Hodges, C. Song and A. Kobayashi, Evaluating the importance of multi-sensory input on memory and the sense of presence in virtual environments, In: Virtual Reality. Proceedings, 1999, IEEE, 222-228, https://doi.org/10.1109/VR.1999.756955, IEEE.

[17] J.A. Gottfried and R.J. Dolan, The nose smells what the eye sees: crossmodal visual facilitation of human olfactory perception, Neuron 39 (2), 2003, 375-386 https://doi.org/10.1016/S0896-6273(03)00392-1.

[18] J.B. Jadauji, J. Djordjevic, J.N. Lundström and C.C. Pack, Modulation of olfactory perception by visual cortex stimulation, J. Neurosci. 32 (9), 2012, 3095-3100 https://doi.org/10.1523/JNEUROSCI.6022-11.2012.

[19] G. Morrot, F. Brochet and D. Dubourdieu, The color of odors, Brain Lang. 79 (2), 2001, 309-320 https://doi.org/10.1006/brln.2001.2493.

[20] S. Kuang and T. Zhang, Smelling directions: olfaction modulates ambiguous visual motion perception, Sci. Rep. 4, 2014, 5796.

[21] G.A. Michael, L. Jacquot, J.L. Millot and G. Brand, Ambient odors modulate visual attentional capture, Neurosci. Lett. 352 (3), 2003, 221-225 https://doi.org/10.1016/j.neulet.2003.08.068.

[22] A.K. Robinson, J. Reinhard and J.B. Mattingley, Olfaction modulates early neural responses to matching visual objects, J. Cognit. Neurosci. 27 (4), 2015, 832-841 https://doi.org/10.1162/jocn_a_00732.

[23] H.S. Seo, E. Roidl, F. Müller and S. Negoias, Odors enhance visual attention to congruent objects, Appetite 54 (3), 2010, 544-549 https://doi.org/10.1016/j.appet.2010.02.011.

[24] R. Michon and J.C. Chebat, The Interaction Effect of Music and Odour on Shopper Spending, Unpublished doctoral dissertation2006, School of Retail Management, Ryerson University; Toronto, Canada.

[25] L. Jiang, M. Masullo and L. Maffei, Effect of odour on multisensory environmental evaluations of road traffic, Environ. Impact Assess. Rev. 60, 2016, 126-133 https://doi.org/10.1016/j.eiar.2016.03.002.

[26] L.M. Anderson, Effects of sounds on preferences for outdoor settings, Environ. Behav. 15 (5), 1983, 539-566 https://doi.org/10.1177/0013916583155001.

[27] J.Y. Jeon, P.J. Lee, J.Y. Hong and D. Cabrera, Non-auditory factors affecting urban soundscape evaluation, J. Acoust. Soc. Am. 130 (6), 2011, 3761 https://doi.org/10.1121/1.3652902.

[28] J.Y. Hong and J.Y. Jeon, Designing sound and visual components for enhancement of urban soundscapes, J. Acoust. Soc. Am. 134 (3), 2013, 2026 https://doi.org/10.1121/1.4817924.

[29] R. Pheasant, K. Horoshenkov, G. Watts and B. Barrett, The acoustic and visual factors influencing the construction of tranquil space in urban and rural environments tranquil spaces-quiet places?, J. Acoust. Soc. Am. 123 (3), 2008, 1446 https://doi.org/10.1121/1.2831735. 
[30] X. Chen, The effect of rose smell on psychological attention function, Theory Research 1 , $2009,162-163$.

[31] J.Y. Hong and Y.J. Jin, The effects of audio-visual factors on perceptions of environmental noise barrier performance, Landsc. Urban Plann. 125 (6), 2014, 28-37 https://doi.org/10.1016/j.landurbplan.2014.02.001.

[32] S.S. Schiffman, B.G. Graham, E.A. Sattelymiller, J. Zervakis and K. Welshbohmer, Taste, smell and neuropsychological performance of individuals at familial risk for Alzheimer's disease, Neurobiol. Aging 23 (3), 2002, 397-404 https://doi.org/10.1016/S0197-4580(01)00337-2.

[33] Y. Pan and Z.H. Meng, Influence of attention on visual and auditory masking effect in condition of visual-auditory dual tasks, ACTA ACUSTUCA 2, 2013, 215-223.

[34] V. Henshaw, Urban Smellscapes: Understanding and Designing City Smell Environments, 2014, Routledge.

[35] J. Pallant, SPSS Survival Manual, 2013, McGraw-Hill Education; UK.

[36] J.D. Porteous and J.F. Mastin, Soundscape, J. Architect. Plann. Res. 2 (3), 1985, 169-186.

[37] A. Tamura, November). An environmental index based on inhabitants' recognition of sounds, In: Proceedings of the 7th International Congress on Noise as a Public Health Problem, 1998, International Commission on Biological Effects of Noise; Sydney, Australia.

[38] J. Kang and M. Zhang, Semantic differential analysis of the soundscape in urban open public spaces, Build. Environ. 45 (1), 2010, 150-157 https://doi.org/10.1016/j.buildenv.2009.05.014.

[39] W. Yang and J. Kang, Acoustic comfort evaluation in urban open public spaces, Appl. Acoust. 66 (2), 2005, 211-229 https://doi.org/10.1016/j.apacoust.2004.07.011.

[40] R.A. Frank and V.D.K. Nj, The contribution of chemosensory factors to individual differences in reported food preferences, Appetite 22 (2), 1994, 101-123 https://doi.org/10.1006/appe.1994.1011.

[41] R.A. Baron, The sweet smell of...helping: effects of pleasant ambient fragrance on prosocial behavior in shopping malls, Pers. Soc. Psychol. Bull. 23 (5), 1997, 498-503 https://doi.org/10.1177/0146167297235005.

[42] X. Zhang, M. Ba, J. Kang and Q. Meng, Effect of soundscape dimensions on acoustic comfort in urban open public spaces, Appl. Acoust. 133, 2018, 73-81 https://doi.org/10.1016/j.apacoust.2017.11.024.

[43] T. Hashimoto and S. Hatano, Effects of Factors Other than Sound to the Perception of Sound Quality, 2001, 17th ICA Rome, CD-ROM.

[44] P. Lercher and B. Schulte-Fortkamp, The relevance of soundscape research to the assessment of noise annoyance at the community level, In: Proceedings of the Eighth International Congress on Noise as a Public Health Problem, 2003, 225-231.

[45] R.E. Apfel and D.R. Raichel, Deaf architects \& blind acousticians? A guide to the principles of sound design, J. Acoust. Soc. Am. 104 (2), 1998, 613 https://doi.org/10.1121/1.423371. 
[46] E. Lange, I. Bishop and I.D. Bishop, (Eds.), Visualization in Landscape and Environmental Planning: Technology and Applications, 2005, Taylor \& Francis.

[47]S.C. Knasko, Pleasant odors and congruency: effects on approach behavior, Chem. Senses 20 (5), 1995, 479-487

[48] J. Xiao, M. Tait and J. Kang, A perceptual model of smellscape pleasantness, Cities, 76, 2018, 105-115 https://doi.org/10.1016/j.cities.2018.01.013

[49] J. Kang and B. Schulte-Fortkamp, Soundscape and the Built Environment, 2016, Taylor \& Francis incorporating Spon, London. https://doi.org/10.1201/b19145

[50] J. Kang, Urban Sound Environment, 2007, Taylor \& Francis incorporating Spon, London. https://doi.org/10.4324/9780203004784

[51] J. Kang, From dBA to soundscape indices: managing our sound environment, Frontiers of Engineering Management, 4(2), 2017, 184-192 https://doi.org/10.15302/j-fem-2017026. 